\title{
DESIGN OF PROTECTIVE STRUCTURES FOR ACTIVE ARCHEOLOGICAL SITES
}

\author{
MILICA D. PETROVIĆ*, ISIDORA D. ILIĆ $\dagger$, NEDA M. DŽOMBIĆ $\dagger$, NENAD D. \\ ŠEKULARAC ${ }^{\dagger}$ \\ ${ }^{1}$ Faculty of Architecture \\ University of Belgrade \\ Bulevar kralja Aleksandra 73/II, 11000 Belgrade, Serbia \\ email: milica.petrovic@arh.bg.ac.rs \\ ${ }^{2}$ Faculty of Architecture \\ University of Belgrade \\ Bulevar kralja Aleksandra 73/II, 11000 Belgrade, Serbia \\ email: isidora@arh.bg.ac.rs \\ email: neda.dzombic@arh.bg.ac.rs \\ email: nenad.sekularac@arh.bg.ac.rs \\ www.arh.bg.ac.rs
}

Key words: structural analysis, design methodology, protective structures, single layer structures

Abstract. This paper presents a structural design methodology in order to find the best possible solution of protective structures for active archeological sites. Besides the typical demands for construction on these sites which arise from the typology and the level of protection of cultural heritage, active archeological sites require a structural solution with regard to time and direction of further excavation. The hypothesis is that modular spatial structures which are easily assembled or disassembled, and have the ability to be extended in one or more directions, are the most functional solution for these sites.

This paper shows the analysis of several types of spatial structures based on the requirements of active archeological sites. It explores the possibilities of structural systems derived from multiplication of one primary element of structure, their material and joints between elements. The most adequate assembly of the entire structure is supposed to be found in the interdependency of the load and the dimension of the element's crosssection, in a suitable geometric configuration. The focus of the analysis is on structures made of short-bearing elements which have the advantage of the efficiency of transport and assembling.

One of the design criteria for this methodology is that the structural system itself looks simple and elegant in order to complement the archeological site. This is shown in the paper through the analysis of patterns in which these structures are assembled. The aim is to correlate the position of the elements in the structural system, the dimensions of cross-section, and the lengths of the straight beams which form the spatial structure in order to find a simple, functional and economical solution for protective structures at active archeological sites.

The conclusion shows the advantages and disadvantages of the design methodology presented in the paper, as well as the discussion about the analyzed spatial structures. This type of methodology that systematizes various design criteria from different fields of research 
engages the problem of design of protective structures for archeological sites and opens up new questions for further research.

\section{INTRODUCTION}

Structures and structural systems formed by multiplication of one primary element of structure are analyzed in this paper. The design of these structures is based on the idea to use short-bearing elements to achieve large spans. By the term long span we assume spans longer than 35 meters, and by short elements we assume beams shorter than 12 meters.

Types of structures presented in this paper were chosen because of their ability to be expanded in multiple directions based on the criteria for design of protective structures in active archeological sites. This paper aims to show the methodology for finding the best possible solution for protective structure that would "grow" together with the archeological site, since the exact time and space span for further excavations is unknown.

It is important to analyze how the joints between elements are made, and the way the elements are positioned within a structure, regardless of scale. Therefore, it is necessary to understand their geometry in order for the design configuration to be favorable to the structural elements. Single layer lattice structures in architecture are the subject of this research, while the theoretical framework is the geometric configuration of structural elements and its effect on structural capacity. The structure is supposed to be easy to assemble and disassemble, without the need for heavy mechanization.

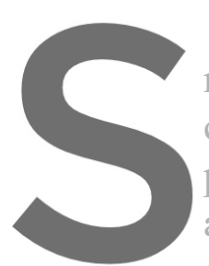

The exploration of

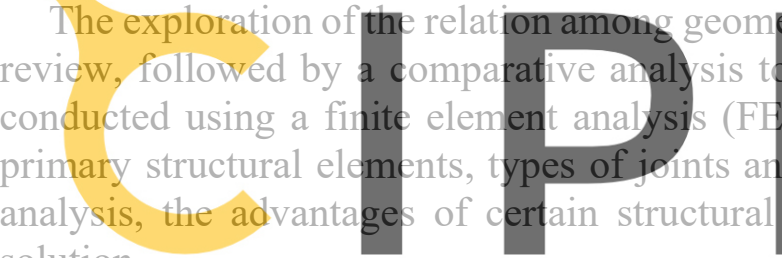

solution.
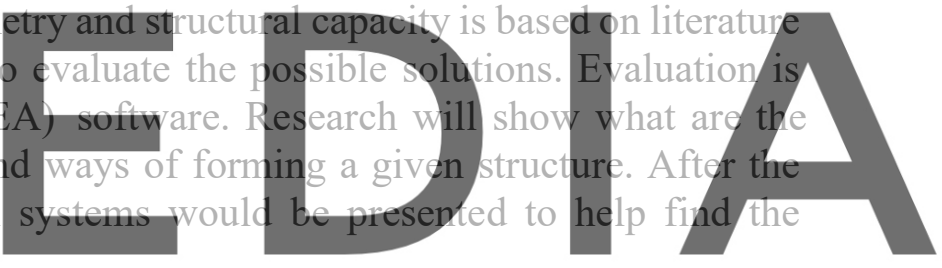

The aim of this research is to find the most efficient configuration of short-bearing structural

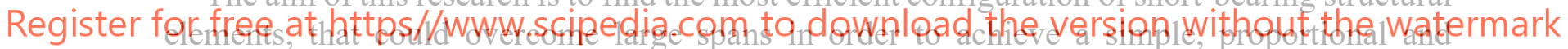

functional structure. The hypothesis is that mathematically consistent pattern would give the adequate geometric configuration of beams that heips in establishing higher efficiency of structure. By designing the minimum dimensions of structural elements, placed in an appropriate pattern, the structure could be perceived as a non-dominant spatial structure, thereby making the archeological site the primary focus of the observer.

\section{ACTIVE ARCHEOLOGICAL SITES}

Archeological sites are places that, by their physical structure, bear witness to the past, or characteristics of the culture that was there. ${ }^{1}$ Active archeological sites are characterized by an unknown direction and time course of the research. In this paper, the term active archeological sites will relate to those sites whose research is still ongoing, which is why it is necessary to enable the protection of already explored parts, as well as those whose research is ongoing. Most often, these archeological sites are large areas, and therefore require major interventions that involve phase development. In the localities explored so far, the emphasis is usually on the most attractive parts, whereby the view of the site as a whole is marginalized. 
Due to its characteristics, it is necessary to find the right type of construction such that it meets all the set requirements. The protective structure should provide a minimum area of reliance, which means that the ideal case would be to have point supports, as well as the unobstructed movement of archeologists - researchers and visitors and the possibility of expansion in accordance with the dynamics of the research. Due to the phase research, and therefore the phase construction of the protective structure, the design of the structure should be conceptually modular, from individual segments that will form a single unique structure. This paper will outline possible types of modular structures for the design of protective structures, their advantages and disadvantages.

Active archeological sites usually cover large areas, entire complexes of objects, often cities or settlements. This implies another criteria for the design of the protective structure - the architectural expression is conditioned by the environment and must not be visually dominant in comparison with archaeological remains.

For a better understanding of the term active archeological sites, an example from Serbia Viminacium, is presented. ${ }^{2}$ The archeological site of the Roman city and military camp occupies an area of 670 ha with a large number of buildings, squares and necropolises. The parts explored so far are the amphitheater, the spa and the northern gate, which form an assembly in the immediate vicinity where the street network of the former city has been identified. This site, as such, has the potential to be protected precisely according to the criteria for the protection of the active site, that is - in phases, with modular structure, in accordance with the dynamics of the research. On the other hand, the protective structure could be designed so that it is large-spaced and dotted, as well as fitting in with the context - both of the formerly built mid-Roman city and of the conternporary appearance of the site.

SINGLE LAYER LATTICH STRUCTURES IN ARCHITECTURV

These structures are gridshells, geodesic domes, free form grid structures, with emphasis on self-supporting structures, such as reciprocal frame structures.

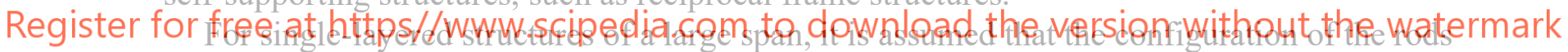
is spatial, the load is uniformly transmitted in all planes of space. This configuration of the rods can be designed such that all structural members are axially stressed, analogous to the shell state. On the other hand, we can design the structure so that the rods are interdependent, and that by creating a hinged or moment connection, they work as a single structural system.

This type of construction is defined as an assembly of short rods that, when connented, can overcome large spans. Also, these structures can be considered lightweight structures, which were among the first to be investigated by Frei Otto and whose experiments gave the basic postulates according to which the above mentioned structures should be designed3:

- Avoidance of the dominant bending effect,

- Transmission of tensile force, low-weight elements, for long spans,

- Transmitting force to short elements, in order to avoid stability problems and large dimensions of supporting structure,

- If it is necessary for pressure to be transmitted over long spans, then they must be integrated as a self-stabilising system,

- Use of adequate cross-sections of the elements in compression in order to avoid static problems. 


\section{Triangulated surfaces}

The triangle, as the basic geometric unit of these structures, is a stable system of articulated rods. The successive extension of two rods and one hinge gives a stable system in a plane or space, thus allowing the formation of a wide variety of geometries that are based on a triangle.

A special type of these structures are geodesic domes, first designed by the architect Buckminster Fuller since 1954. In order to maximize the potential of steel and its ability to accept tension forces well, Fuller formed a dome from a series of rods that follow geodesic lines. Geodesic line is the shortest distance between two points on a curved surface, which can be approximated to a straight line. When he patented his first geodesic dome, he pointed out that his dome was as wide as the Pantheon, but a hundred times lighter. ${ }^{4}$ This architect saw the geometric characteristics of a triangle, as a stable system, and applied them to form the surface of a sphere. In this way, a stable structural system was realized, with the stress state being analogous to reinforced concrete shells, therefore, axial forces occur in all the rods. ${ }^{5}$ The postulate on which Fuller's geodesic domes are based is: "continuous tension - discontinuous pressure". ${ }^{6}$ It can be clearly seen from this that each rod, which is approximately horizontal, is in tension. These rods form irregular rings of geodesic polyhedron and as such form the discontinuity of the compressive forces in the rest of the rods.

Buckminster Fuller reduced the sphere to a system of triangles and thus formed a stable structural system. On the other hand, the sphere has the largest volume relative to the surface of the envelope. From an architectural design point of view, this means that a maximum range

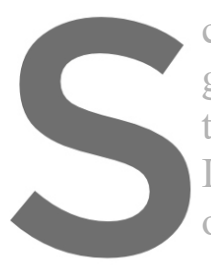
can be achieved with minimal material consumption. Related t
geometry of the sphere to form structural elements arises. Ar
the sphere to polyhedron, and the curyed meridians and paralle
In this way it was possible to retain all the advantages arising fron
of the sphere as a geometric body, but also of the triangle as a

The modular elements that make up the geodesic domes - the joints and rods of the same dimensions represent their greatest advantage. Due to the even distribution of forces in the

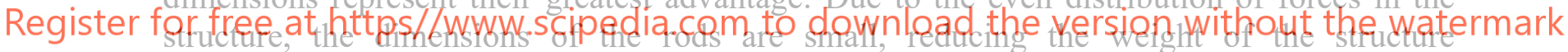

significantly. The advantages of these domes are reflected in their widespread use in practice.

Miost often they are made of steel or wooden rods connected by steel ties. Point supports, along the perimeter of the dome, fulfill one of the criteria for the design of structures in active archeological sites, however, very few sites have circular shaped objects in floor plan.

Mesh surfaces are evolving in simultaneously with technological progress. These surfaces emerged as a continuation of exploration of shells of non-Euclidean geometries. By extending the Gaussian curves to form complex curves in two or more directions, a free-form emerges which is much more complex for structural design and analysis.

They are often designed in a way that each element of the structure is continuous, or superimposed, which further implies that the organization of the structure must be very well composed, without improvisation, due to the fact that every element corresponds to only one position within the structure.

Also, we can consider these surfaces as a continuation of the study of triangulated surfaces, as the curvature of any structure is based on a triangle.

Through generative software, designing and looking at these structures has become simpler and more efficient. The key advantages of these structures are reflected in the placement of the 
supporting points so that they correspond as closely as possible to the remains of an active archaeological site, without restriction. Free forms can be constructed in any material, which is an added advantage.

\section{Reciprocal frame structures}

The principle of reciprocal structures, mostly formed of wooden rods, dates back to the Early Stone Age, and is based on the mutual carrying rods in order to bridge a span. The lengths of the rods vary with the dimensions of the base and the pattern of the reciprocal structure itself.

The principle underlying these structures is based on the temporarily supported first element of the structure, with each subsequent beam relying on the previous one, while the last beam placed will carry the first one. In this way, a closed cyclic movement of the force in the structure is formed, which enables each element of the structure to be both load bearing and carried. Therefore, each element accepts the load equally, so it has the same structural forces. Patterns in which these structures can be formed are based on the rotation of the structural elements. Such patterns are formed over the basics of various polygons, with the number of sides of the polygon depending on the number of rods in the structure, that is, the angle of rotation of each element. $^{7}$

Due to the possibility of combining several different configurations (e.g. triangle hexagonal), the floor plan on which this construction is designed today does not have to be the basic geometry. Namely, the greatest advantage of these constructions is the prefabrication, that

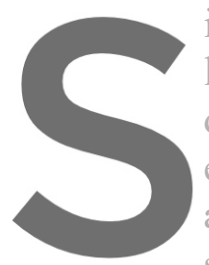
is, no matter how much length, with a possible designed in a large nu elements of these struct as a notch joint, i.e., the groor section's height. Using change
mber of
tures now
groove
the notc enstruction volum
fin patterns, with a
wadays a e usual ume is changed
alms of cross-s
ally made of ste
nents by sealin
sembly process
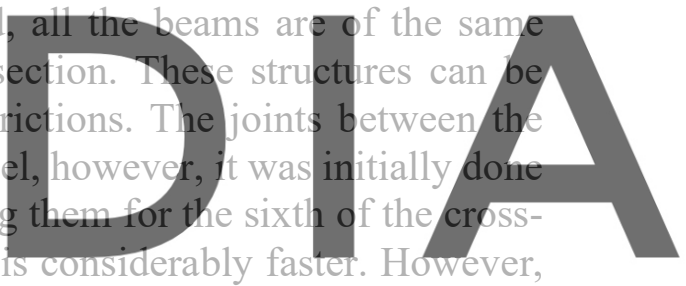
due to the necessity of reducing static height of the cross-section by the use of a notch joint, a

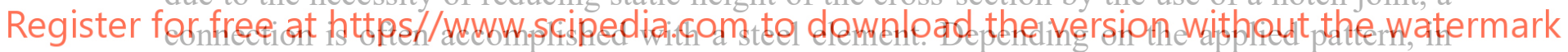
reciprocal frame structures, the length of each element varies, as well as the way in which the connection between two or more elements is achieved. The dimensions of the cross-section can vary with the span, as well as the load capacity of the structure. ${ }^{8}$

Architect Friedrich Zollinger patented Zollinger's first construction in 1910. The construction consists of segments of solid wooden carriers, about 2 meters long, of uniform geometry. In the literature, Zollinger's construction is called Lamella roof, according to author Martin Tamke. ${ }^{9}$ By the term lamella, one repetitive segment is considered, which makes this construction, so this term can be translated as a construction of the same segments. The principle by which this construction functions is such that at the endpoints of two beams of one direction there are joints to the centre of the beam from the other direction. In this way, the interlocking of the elements was achieved, resulting in a span of up to 40 meters. The pattern by which the elements are arranged, although not listed in the analyzed set of patterns, is the projection of rhombus on the roof surface. The original Zollinger construction implies the geometry of the pointed arc, which for the first time appears as a stylistic feature of Gothic architecture. $^{10}$ 
The connections of wooden carriers were articulated, which was the reason for further research and improvement of these structures. Hugo Junkers, a mechanical engineer and aircraft designer, patented in 1924 a similar construction that consisted only of steel elements among which moment connections were formed. He found inspiration in the design of the aircraft itself. ${ }^{11}$

The Zollinger construction is very similar to reciprocal constructions according to the load transfer principle. The advantages of this construction are the simplicity and the efficiency of assembling, as well as the prefabrication of elements. A disadvantage of this construction is a geometry of floor plan on which these structures are projected, which is predominantly rectangular. In case it is necessary to use the principle of this structure for more complex geometry, each connection of the two elements should be specifically designed due to differences in the spatial orientation of the connections themselves. Therefore, each rod should have been separately designed, which would result in neglected prefabrication benefits.

\section{Comparison of structural systems}

Although geodesic domes, as representatives of regular triangulated surfaces, have the same elements as well as typical connection details, with the goal to achieve the largest possible span with the least material used, their design constraints are counteracted by the criteria that the design of structure over an active archeological site must have as flexible positions of support points as possible.

\section{On the other hand}

supporting points, is structures in the function

a strong architectural

sites. Also, these structur

elements and a complex assentity process.
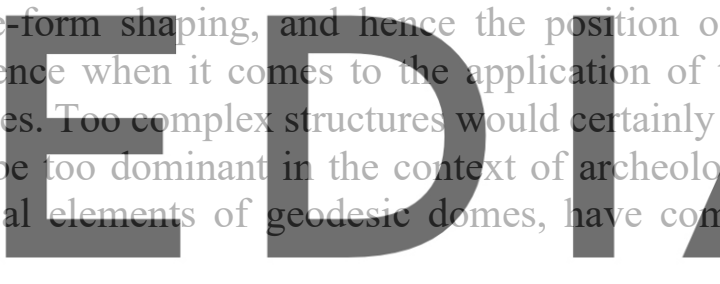

However, reciprocal structures, as an example of free-form modular structures, are the most

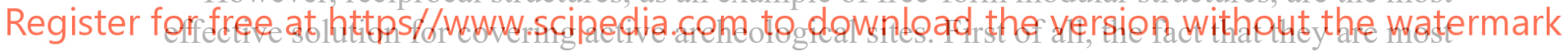

often constructed in wood as an applied material, which equally accepts pressure and tension

and it is also the lightest structural material. Therefore, these constructions are of high loadbearing capacity and of low weight compared to other structural materials.

With the use of wood, these structures easily fit into surrounding context, especially due to the fact that active archeological sites are often located outside the built environment, and the newly designed structure should be integrated with the landscape in which it is located. The load-bearing capacity of reciprocal structures is of the utmost importance because each element of the structure is equally loaded or the load is gradually increased, without extreme changes in load transfer. Also, the assembling of reciprocal structures, as a structure consisting of short rods, is one of the key advantages of why the reciprocal structures are suitable for covering part of the archaeological site or for the phased development of the archaeological site, and therefore the protective structure.

Reciprocal structures can also be seen as modular structures, point-supported, simply interconnected (groove joinery or by screw), or as a combination of identical elements of geodesic domes and flexibility of free forms. 


\section{INTERDEPENDENCE OF PATTERNS, LOAD-BEARING AND DIMENSIONS OF CROSS-SECTIONS OF RECIPROCAL FRAME STRUCTURES}

Patterns shown in the first iteration represent two-dimensional configuration made of short rods. The structural forces of the first five patterns, which were derived from basic geometric figures, or a combination of various regular polygons, were analyzed. In the first iteration, precisely because it was a two-dimensional configuration, it was necessary to evaluate the patterns through the deflection they cause. The first check was made using a program in order to obtain the expected value of the deflection. The assumed cross-section of the beam is $20 / 60$ $\mathrm{cm}$, the input load is $2.92 \mathrm{kN} / \mathrm{m}$ ' (derived from the analysis of the load of the roof cover and the own weight of the beam), and the span of this girder is 20 meters. This deflection which will be compared to the deflection of other patterns is obtained from calculation of this beam, and its value is $5.63 \mathrm{~cm}$.

The patterns were formed from a system of short rods, which are rigidly connected, and then analyzed in software. Two sets of patterns were evaluated simultaneously, which differ in the way they are supported. The first set of patterns (in the table labelled T) is supported only in the vertices of the square (point supports) while the second set (in the table labelled with L) is supported by the edge of the square field, that is, in a linear assembly of point supports. It can be assumed that the second set will give better results. Each constructive field is formed over a square base that is $20 \times 20$ meters.
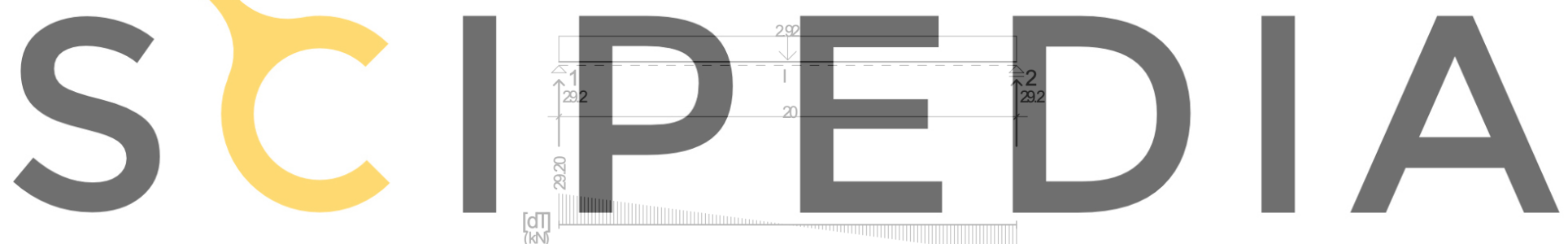

Register for free at https//www.scipedia.com to download the version without the watermark

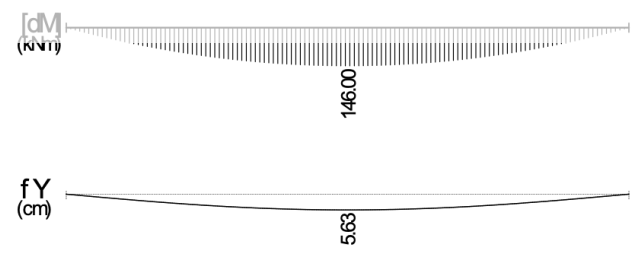

Figure 1: Static analysis of a simply supported beam of $20 \mathrm{~m}$ span

Patterns are named according to the geometry from which they were derived, but now they will be given numerical labels: orthogonal matrix (1), diagonal matrix (2), triangulated matrix (3), hexagonal matrix (4) and matrix hexagonal triangle (5). As it was already mentioned, the 
matrices are formed so that each geometry forms a field of approximately same surface, so that the linear load applied to each girder is approximately of the same intensity.
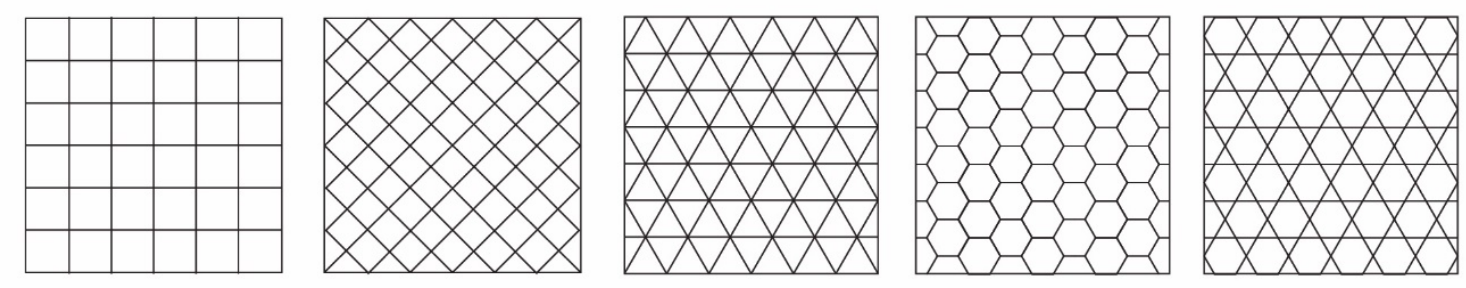

Representation of deformed models:

orthogonal matrix (1)

diagonal matrix (2)

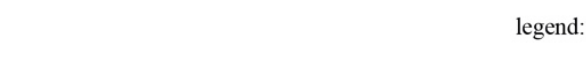

triangulated matrix (3)

hexagonal matrix (4) matrix hexagonal triangle (5)
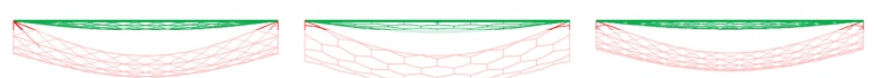

\begin{tabular}{|l|l|l|l|l|l|l|l|l|l|l|}
\hline $\mathrm{Zp}$ & deformation in $\mathrm{m} / 1000$ & rank & deformation in $\mathrm{m} / 1000$ & rank & deformation in $\mathrm{m} / 1000$ & rank & deformation in $\mathrm{m} / 1000$ & rank & deformation in $\mathrm{m} / 1000$ & rank \\
\hline
\end{tabular} \begin{tabular}{l|r}
\hline $\mathrm{T}$ & 748 \\
\hline
\end{tabular}

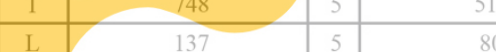

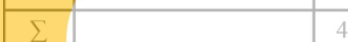

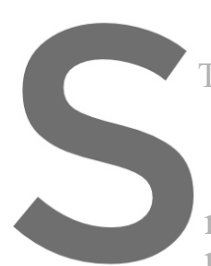

Table 1: Deflection comparison with evaluation-basic patterns
The first comparative analysis of both sets was done throu
model, where it was evident that the set of matrices with sup had significantly largerdeflections than the linear set.

At first point, it seemed that the diagonal matrix (hereinafter: matrix 2) had the smallest

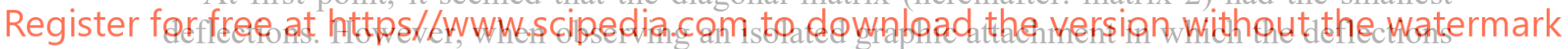

of the first and second set are seen, on the basis of the more drastic differences in the deflections

in the set of point-oriented structure, one can observe which configurations have the most favourable behaviour in the plane.

Table 1 shows the deflections of analyzed patterns in two sets. Set 1, marked with the letter $\mathrm{T}$, represents a support in the vertices of the square, while the set 2, marked with the letter $\mathrm{L}$, represents a linear support along the sides of the square base. All the analyzed patterns were shown in the table, whereupon the maximum deflections for each were drawn. Of the five analyzed, the three patterns, according to the estimation are of the same rank, regardless of the type of reliance, however, two patterns are best rated, so the triangulated matrix has less than the matrices of the hexagonal triangle when leaning point in the square of the square. On the other hand, despite small differences, matrix no. 5 has smaller deflections than matrix no. 3, if it relies on a liner support.

Although these two matrices proved to be the best in numerical value analysis, the diagonal matrix is very close to results. Another similarity, when analyzing two of the best patterns, is their relevant angle when forming the pattern itself. Both matrices in their configurations have a triangle, or an angle of $60^{\circ}$. Which leads to the question of whether the diagonal matrix is 
reduced if it is formed using the same angle of $60^{\circ}$ instead of the $45^{\circ}$, angle at which it is currently. However, regardless of this assumption, the diagonal matrix deflections, if formed using an angle of $60^{\circ}$, is $10.3 \mathrm{~cm}$, which is more than the first configuration with an angle of $45^{\circ}$.

Due to the obtained results, through the following analysis, or analysis of the geometry of the patterns that form reciprocal frame structures, these two configurations will be evaluated again as the best in the plane. It is extremely advantageous that these two matrices have proved to be the best, due to the various possibilities of combining the triangular and hexagonal configuration of reciprocal structures. Also, these configurations of reciprocal modules of the construction give the possibility of creating spatial single layer structures.

However, the biggest problem due to this analysis is the assumption that all the beams are connected using a moment connection. This can be a problem for any applied material, the detail of the connection will have to be formed in such a way that it transmits the bending moments from one structural element to the next. When the steel is the assumed structural material, this connection can be achieved by welding the girders one to another, but due to the application of short elements the assembling of the construction would take too long. However, in the case of short wooden elements, a special type of connection needs to be applied, achieved by a steel element that could transfer the bending moments.
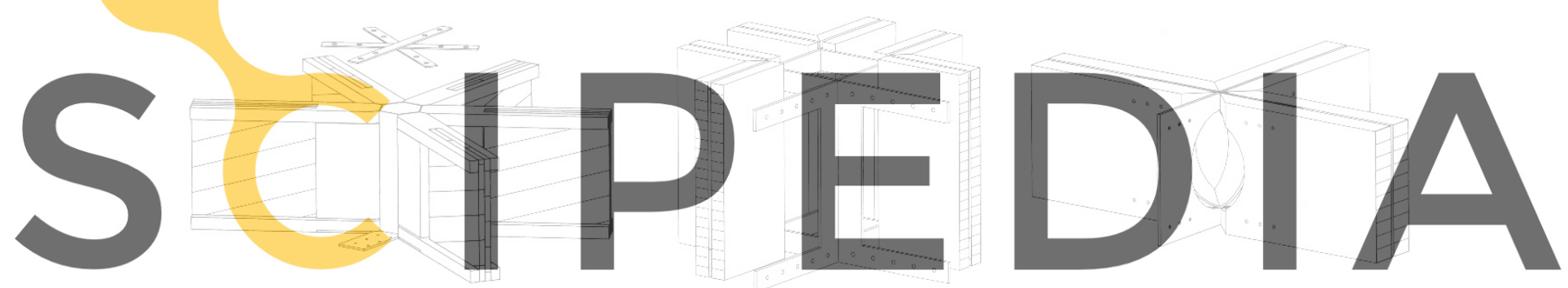

Register for free at https//www.scipedia.com to download the version without the watermark

By analyzing the matrices of reciprocal frame structures, as expected, bigger defiections were obtained, due to the hinged connections between the elements. However, when talking about the detail of the connection in wooden structures, it can be done as a notch joint, or with a reinforcement of the steel elements. The possibility of making a notch connection would only be valid for spans up to 12 meters, while the reinforcement of the connection would apply for spans larger than 12 meters. This feature of reciprocal frame structures enables easy and efficient assembling on site. That is, the mounting process would be considerably faster, and therefore cheaper, compared to achieving a rigid connection. Software analysis of patterns that 
form reciprocal structures in the plane, where the joints of the girders were done as hinged connections, gave the following results when it comes to deflection of the model.

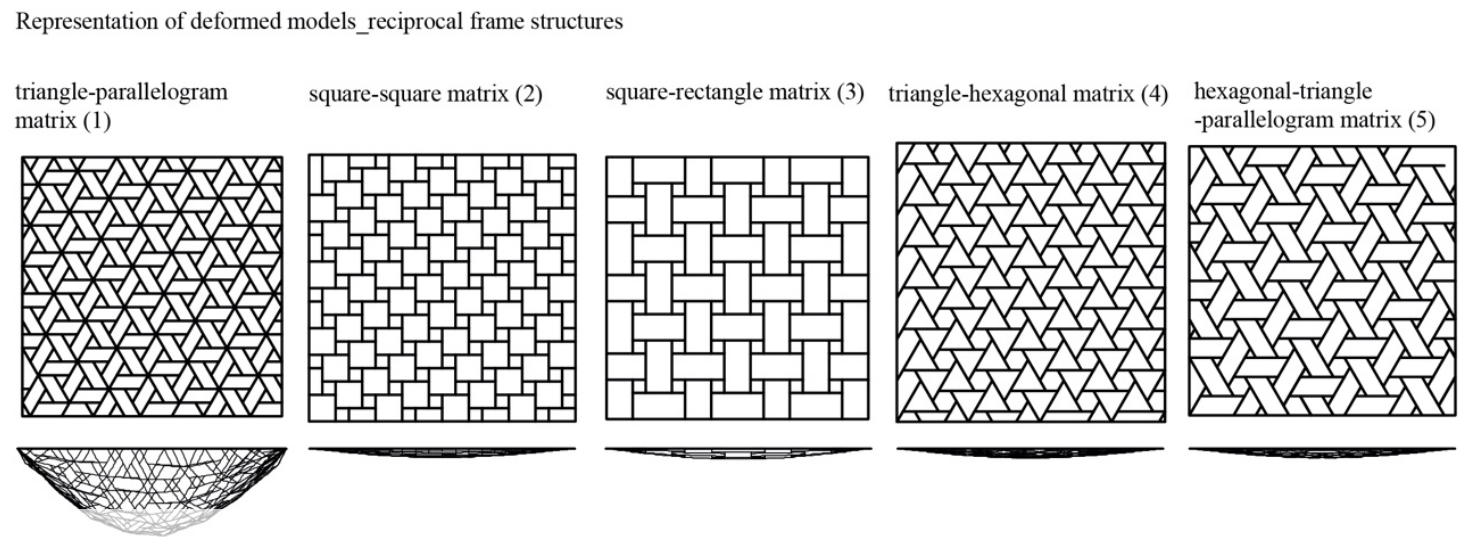

\begin{tabular}{|c|c|c|c|c|c|c|c|c|c|c|}
\hline $\mathrm{Zp}$ & deformation in $\mathrm{m} / 1000$ & rank & deformation in $\mathrm{m} / 1000$ & rank & deformation in $\mathrm{m} / 1000$ & rank & deformation in $\mathrm{m} / 1000$ & rank & deformation in $\mathrm{m} / 1000$ & rank \\
\hline $\mathrm{L}$ & 1290 & 5 & 144 & 1 & 198 & 4 & 168 & 3 & 165 & 2 \\
\hline$\Sigma$ & & 5 & & 1 & & 4 & & 3 & & 2 \\
\hline
\end{tabular}

value 5 - worst rated, value 1 - best rated
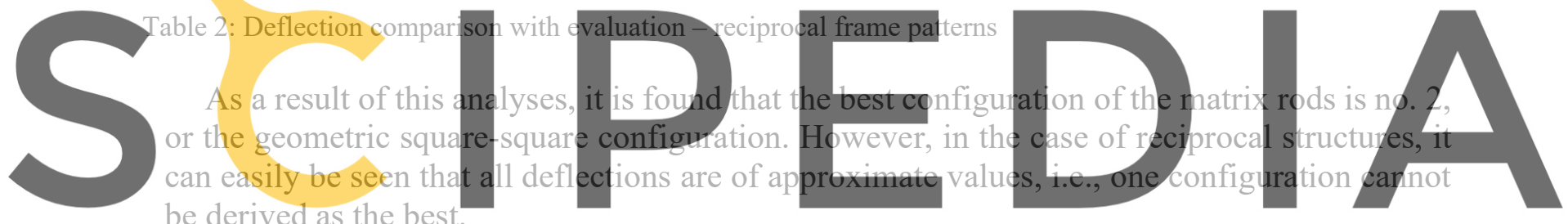
be derived as the best.

Bearing in mind the efficiency of connecting and easy assembling. of reciprocal structures,

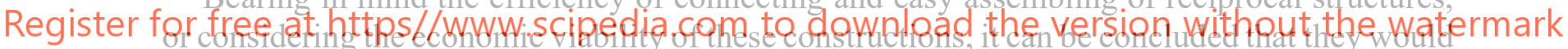

be significantly more applicable than patterns with rigid connections. Namely, using wood, as a natural material, as well as reducing the use of steel in the construction, can affect the reduction of carbon dioxide in the atmosphere precisely because of the reduction of $\mathrm{CO} 2$ emissions from the exploitation of steel. In this way, a green, economically profitable construction is obtained, which as such can be applied in different locations of specific construction conditions, such as archaeological sites.

It is necessary to note that in this chapter only two-dimensional configurations of the girders were analyzed, i.e. that considerably better static characteristics are expected by using single or double curvature.

\section{CONCLUSION}

This design approach for choosing the appropriate structural system is based on criteria set by the characteristics for active archeological sites. The analysis of interdependency between structural pattern and the dimensions of beam cross-section are in direct correlation to the structural form of the protective structure and its overall appearance in the archeological 
landscape. "The good building is not the one that hurts the landscape, but one which makes the landscape more beautiful than it was before the building was built." 12

Other criteria in this methodology is guided by the position of the observer, because the human eye searches for natural shapes and structures in its environment that hold a sense in itself. ${ }^{13}$ Therefore, the chosen bearing structure has to be simple and logical to a careful eye, embracing the surrounding landscape.

Reciprocal frame structures are modular, usually made of short wooden beams, connected with simple joints, making them simple for construction, with no need for heavy mechanization to and on site, thus fulfilling the criteria for active archeological sites. These structures are ecological, fitting perfectly in the natural surroundings. The most appropriate pattern for this structure is the one made of larger and smaller squares, which can be easily modified in order to be expanded. This light structure has small scale point supports that would not damage the remains on site. They are also very elegant, so they do not turn away the attention of the observer from the site.

The presented methodology is based on specific criteria for design of protective structures for active archeological sites in order to find the best possible solution, but it could be improved with case studies oriented towards specific sites. Model analysis on different types of structures could complete this methodology, so it can be used in practice, as well as in theoretical approach.

\section{REFERENCES}

[1] Cambridge University Press. Cambrigde Dictionary. Retrieved from Meaning of archaeology in English, (2020, January 31): https://dictionary.cambridge.org/dictionary/english/archaeology

[2] Nikolić, E. Evaluation of the protection and presentation of historic buildings in the Viminacium Archaeological Park in relation to their spatial context. Spatium, (2018), pp. 26-37.

[3] Nerdinger, W., Meissner, I., Möller, E., \& Grđanski, M. Frei Otto Complete Works: Lightweight construction - natural design. Basel: Birkhäuser, (2005)

[4] Fuller, R. B., Inventions - The patented works of R. Buckminster Fuller. New York: St. Martin's press, (1983), pp. 129.

[5] United States of America Patent No. 2,682,235, 1954

[6] Fuller, R. B., \& Applewhite, E. Synergetics. Sebastopol: Macmillan Publishing Co. Inc, $(1975,1979)$

[7] Popovic Larsen, O. Reciprocal Frame Architecture. Oxford: Elsevier Ltd, (2003)

[8] Song, P., Fu, C.-W., Goswami, P., Zheng, J., Mitra, N. J., \& Cohen-Or, D. An Interactive Computational Design Tool for Large. Nexus Network Journal, (2014) pp. 109-118.

[9] Tamke, M., Rüber, J., \& Jungjohanne, H. Lamella Flock. In C. Ceccato, L. Hesselgren, M. Pauly, H. Pottmann, \& J. Wallner, Advances in Architectural Geometry 2010, Vienna: Springer. (2010), pp. 37-48

[10] Ceccato, C., Hesselgren, L., Pauly, M., Pottmann, H., \& Wallner, J. Advances in Architectural Geometry 2010. Vienna: Springer. (2010) 
[11] Weller, B. Lamella Roof Constructions by Hugo Junkers. Evolution and Trends in Design, Analysis and Construction of Shell and Spatial Structures,

Valencia: Universidad Politecnica de Valencia. (2009), pp. 1611-1621

[12] Hertzberg, M., \& Lloyd Wright, F. Wright in Racine: The Architect's Vision for One American City. San Francisco: Pomegranate, (2004)

[13] Balz, M. Phantasy in space. An Anthology of Structural Morfology, (2009), pp. 112. 